\title{
Automatic Segmentation Based on Statistical Parameters for Infrared Images
}

\author{
D.O.I - 10.51201/Jusst12567 \\ http://doi.org/10.51201/Jusst12567
}

\begin{abstract}
Akshay Isalkar and Manikandan K.
School of Computing Science and Engineering, VIT Bhopal University, India.
\end{abstract}

\begin{abstract}
Image segmentation is an integral part in recognizing pat-terns. Image segmentation techniques aim to partition of images into several parts so that object and background are separated for image un-derstanding and analysis. There are many image segmentation method presented but very few work with infrared images (IR). Fast improving performance and falling cost of IR sensors strengthen IR image process-ing popular. IR images provide more capability to capture images at large distance without light illumination in diverse environment condi-tions, which is not present in visual images. IR image segmentation grows slowly in practical aspect rather than theoretical. Thresholding is sim-ple and widely used method for image segmentation. For this work, IR images are captured using SeekThermal IR sensor. The various statis-tical parameters such as mean, mode, median, standard deviation (SD) etc. are retrieved from input image. Based on these statistical parame-ters a new automatic method for image segmentation is proposed called as StatSDM. The proposed StatSDM method uses combination SD and median for automatic image thresholding. The performance of statSDM is evaluated with standard statistical based image segmentation meth-ods. The results are compared with global Ostu, Max Entropy, Trian-gle and Percentile thresholding techniques show promising performance. This work presents automatic and efficient thresholding method for IR image segmentation.
\end{abstract}

Keywords: Infrared Image Automatic Thresholding Ostu Image Segmentation

\section{Introduction}

According to NASA, $68 \%$ of the Universe is dark energy and we cannot elude such amount of dark energy. Whenever darkness overcomes we look hopefully to-wards Infrared (IR) sensors. IR imaging technology forms an image scene based on IR radiations emitted by hot/cold objects. It offers capabilities not available with visible imaging, such as capturing images in dark, measuring object's tem-perature and so on. Over past few years, fast improving performance and falling prices of IR sensors have spurred rapid expansion in the use of IR sensors. Object 
detection in IR imaging is attracting great interest in variety of fields. Object detection involves detecting instances from particular class in an image.

Earlier object detection in IR imaging has four major tasks of preprocessing, detection, segmentation and classification [9][13] . Object detection methods has roughly classified into two types. Sense earlier to track and track previous to Sense [14]. It is a long term goal in IR image processing to detect generic objects automatically. Also due to cluttered background, variation in object, changing object pose and hot background than object, automatic detection persists to be a challenging problem [8]. Therefore the main issue is how objet detection is automatic in IR images for all generic objects.

In order to set this work into object segmentation context, it is worth reviewing some of the specialized thresholding methods like Ostu, MaxEntropy, Triangle, and Percentile. Automatic thresholding for object detection in visi-ble images grow faster than IR imaging [8]. Number of thresholding methods available that are applicable to visual images and show significant results.

This work proposes a simple and automatic thresholding method StatSDM based on combination of Median and Standard Deviation (SD) for object detection in IR imaging. To make this method automatic, statistical parameters from an image are retrieved and set proper combination of Median and SD to achieve thresholding. This technique of automatic object detection has some advantages; it does not depend on temperature dissimilarity and can be functional in all circumstances such as object inconsistency and warm surroundings than object. Also, it is free from difficult and complicated methods, which desires more time for processing. It is particularly valuable in detecting generic object.

This paper is planned as follows: first review of thresholding methods under Image Segmentation is discussed in section 2. Then image acquisition and preprocessing followed by thresholding algorithms in broad are describe in section 3 . In section 4, experiments to test performance of algorithms. Section 5 concludes paper with future scope.

\section{IR Image Segmentation}

Segmentation of IR Image is one of the promising issues associated with anal-ysis of images. The segmentation on image is performed to tackle interested object.

The segmentation is performed on image using pixel-based or object-based properties such as shape, edges, size, texture, orientation and intensity variation

[3][12]. Segmentation process is performed with two goals. The first aims to subdivide an image for object analysis while second for faster analysis by changing image representation. A single or mixture of segmentation methods can be used to achieve the segmentation goals according to applications [3][4][5]. The thresholding based segmentation; edge segmentation and area segmenta-tion are the three types of segmentation techniques. There are extensive varieties of image segmentation methods have been suggested. Even though there is no unique

standard image segmentation way that can applicable to all kinds of gen-eral images with effective results [5][8]. The image segmentation goals fluctuate 
according to application and type of image dataset. Applications of different segmentation algorithms based of diverse assumption about the type of images [5].

Image thresholding separate out parts of an image analogous to objects which we desire to study. This separation is based on the dissimilarity of intensity values connecting the object pixels and the environment pixels. It is straightforward and easy for implementation therefore it is widely used in different segmentation applications [1][6]. Let IR(x,y) be the original IR image then after threshold, a binary image $\operatorname{IRB}(\mathrm{x}, \mathrm{y})$ is obtained by using equation 1 .

$$
\operatorname{IRB}(x, y)= \begin{cases}1, & \text { if } \operatorname{IR}(x, y)>\mathrm{Th} . \\ 0, & \text { otherwise }\end{cases}
$$

Where Th represents the threshold value. The resulting $\operatorname{IRB}(\mathrm{x}, \mathrm{y})$ is binary image with 1 as object and 0 as background.

Human eyes can easily distinguish between object and background. Whereas, image thresholding is a difficult task to separate object from surroundings. For developing image thresholding algorithms, a grey level histogram is considered as an efficient tool [2].

Let us consider $\operatorname{IR}(\mathrm{x}, \mathrm{y})$ be raw infrared image with gray level intensity $\mathrm{L}$ ranging from 0 to $(\mathrm{L}-1)$. The probability of every gray-level pair $\mathrm{p}(\mathrm{x}, \mathrm{y})$ is calculated by 2 .

$$
P(x, y)=\frac{f(x, y)}{M \times N}
$$

And we know, $0 \leq p(x, y) \leq 1$

Therefore,

$$
\sum_{x=0}^{L-1} \sum_{y=0}^{L-1} p(x, y)=1
$$

Where frequency pair of $(x, y)$ is $f(x, y)$. $M$ and $N$ denotes number of pixels in rows and columns of IR image respectively. The histogram is constructed as two dimensional gray histogram supported on pairs of gray-level frequencies [6].

\subsection{Ostu Method}

Ostu[4][15] proposed a popular thresholding method widely used for document processing. This method is approximately one dimensional and discrete Fisher's Discriminant function. It assumes two classes: object pixels and non-object pixels. It calculates optimal threshold untying two classes such that intra classvariance is minimum [4][14].

Consider L gray-levels of IR image with normalized histogram, $\mathrm{P}(\mathrm{k})$ is normalized frequency of $\mathrm{k}$, for all gray-level value $\mathrm{k}$. Considering that we have put the threshold at $\mathrm{T}$. The probability of background and object will be given by equations 4 and 5 respectively. 


$$
\begin{gathered}
q_{b}^{T}=\sum_{k=1}^{T} P(k) \\
q_{o}^{T}=\sum_{k=T+1}^{L} P(k)=1-q_{b}^{T}
\end{gathered}
$$

The mean gray level value associated with background and object will be calculated as in equations 6 and 7 respectively.

$$
\begin{gathered}
\mu_{b}^{T}=\frac{\sum_{k=1}^{T} k P(k)}{\sum_{k=1}^{T} P(k)}=\frac{\sum_{k=1}^{T} k P(k)}{q_{b}^{T}} \\
\mu_{o}^{T}=\frac{\sum_{k=T+1}^{L} k P(k)}{\sum_{k=T+1}^{L} P(k)}=\frac{\sum_{k=T+1}^{L} k P(k)}{q_{o}^{T}}
\end{gathered}
$$

The mean value of gray-level for entire image is given by equation8.

$$
\mu=\mu_{b}^{T}+\mu_{o}^{T}=\frac{\sum_{k=1}^{L} k P(k)}{\sum_{k=1}^{L} P(k)}=\sum_{k=1}^{L} k P(k)
$$

The variance for the background and object is considered as in equations 9 and 10 .

$$
\begin{gathered}
\operatorname{var}_{b}^{T}=\frac{\left.\sum_{k=1}^{T}\left(k-\mu_{b}^{T}\right)^{2} P(k)\right)}{\sum_{k=1}^{T} P(k)}=\frac{1}{q_{b}^{T}} \sum_{k=1}^{T}\left(k-\mu_{b}^{T}\right)^{2} P(k) \\
\operatorname{var}_{o}^{T}=\frac{\left.\sum_{k=T+1}^{L}\left(k-\mu_{o}^{T}\right)^{2} P(k)\right)}{\sum_{k=T+1}^{L} P(k)}=\frac{1}{q_{o}^{T}} \sum_{k=T+1}^{L}\left(k-\mu_{o}^{T}\right)^{2} P(k)
\end{gathered}
$$

The variance of whole image is given by equation 11 below

$$
v a r=\operatorname{var}_{b}^{T}+\operatorname{var}_{o}^{T}=\sum_{k=1}^{L}(k-\mu)^{2} P(k)
$$

Within class variance is given by equation 12

$$
\operatorname{var}(\text { within }- \text { class })=q_{b}^{T} * \operatorname{var}_{b}^{T}+q_{o}^{T} * \operatorname{var}_{o}^{T}
$$

The optimal value of threshold for Ostu method is selected by minimizing within class variance.

By small modification between class variance is calculated and far quicker to calculate. The threshold with maximum between class variance is selected as optimal value of threshold for Ostu metho das per equation 13.

$$
\operatorname{var}(\text { between }- \text { class })=q_{b}^{T} * q_{o}^{T}\left(\mu_{b}^{T}-\mu_{o}^{T}\right)^{2}
$$

Ostu method does not perform well with patchy illumination and assume bimodal (two classes) histogram of image. 


\subsection{MaxEntropy Method}

Another optimal thresholding method MaxEntropy is implemented Kapur-SahooWong[4][16]. Maximum Entropy thresholding method is subjected to maximum inter-class entropy [1][2]].

Let $\mathrm{P}(\mathrm{T})$ defines cumulative probability and it is represented by 14 .

$$
P(T)=\sum_{k=0}^{T} p(k)
$$

We know equation 15.

$$
p(k)=\frac{n_{k}}{N}
$$

Where $\mathrm{p}(\mathrm{k})$ is the probability distribution of histogram, $n_{k}$ is the level $\mathrm{k}$ of frequency and $\mathrm{N}$ is image size. The optimal threshold for MaxEntropy is maximum sum of entropies for background and object given by 16 .

$$
T=\arg \left\{\max 1 \leq T \leq L\left(E_{b}^{T}+E_{o}^{T}\right)\right\}
$$

Where, $E_{b}^{T}$ and $E_{o}^{T}$ is given by equations 17 and 18 respectively.

$$
\begin{gathered}
E_{b}^{T}=-\sum_{k=1}^{T} \frac{p(k)}{p(T)} \ln \left(\frac{p(k)}{p(T)}\right) \\
E_{o}^{T}=-\sum_{k=T+1}^{L} \frac{p(k)}{p(T)} \ln \left(\frac{p(k)}{p(T)}\right)
\end{gathered}
$$

Where, $\mathrm{L}$ is maximum gray level value in an image. $E_{b}^{T}$ and $E_{o}^{T}$ are background and foreground image entropies respectively.

\subsection{StatSDM Method}

In this method, automatic thresholding is acheived by selecting sum of median and standard deviation (StD)of input IR image. Median is the middle gray-value of ordered image data. The stsndard deviation is square root of the variance (Var) form above equation.

\section{Methodology}

The proposed method uses combination of median and standard deviation to set optimal threshold. The flowchart of working is shown in figure[1]. The acquired input IR images have noise. To remove noise the median filter is applied in preprocessing step. After statistical parameters such as Mean, Median, Standard Deviation, Min, Max etc. has been recorded[17]. Thresholding for segmentation to mark object is carried out with sum of median and standard deviation. Region of interest (ROI) is extracted from IR image and statistical parameters are recorded from extracted ROI to evaluate efficiency. The efficient algorithm is selected for final segmentation. 


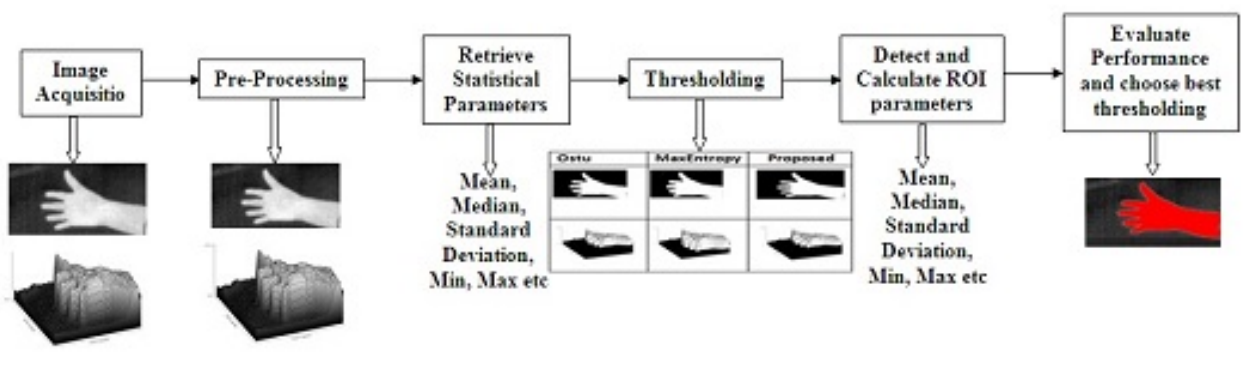

Fig. 1. Flowchart of proposed work.

\subsection{Algorithm}

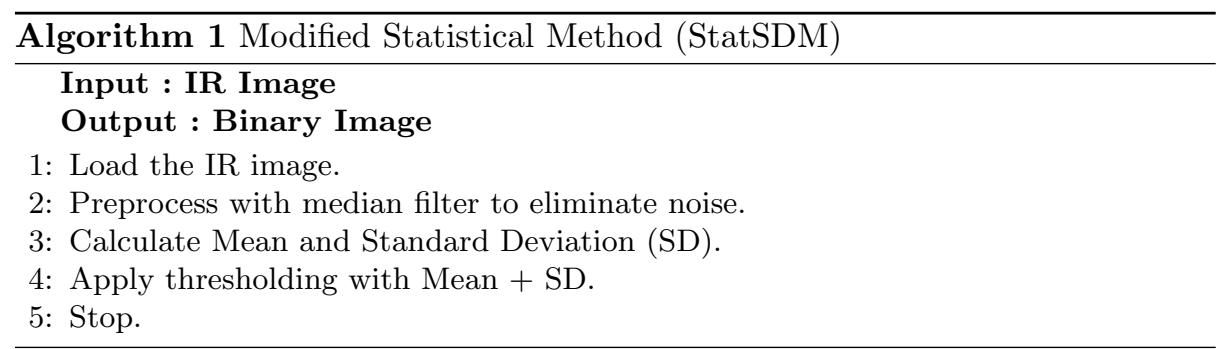

\section{Result}

The IR images are acquired from the $206 \times 156$ IR SeekThermal camera with $12 \mu$ Pixel Pitch and $360^{\circ}$ fields of view. The dataset consist of IR general objects such as Animals, Humans and Objects with total count of 51 images. The proposed algorithm is compared with global Ostu and Max-Entropy thresholding methods. Threshold values for Ostu method and Max Entropy method is calculated by ImageJ tool whereas threshold values for proposed method is calculated using OpenCV Java library using Eclipse. Following table 1 shows the different threshold values using Ostu method, Max Entropy method and proposes modified statistical method for sample images.

\subsection{Evaluation Parameters}

Recall and precision are main parameters to evaluate performance of image segmentation [2][19]. Precision is defined as the number of object pixels detected by the method divided by total number of pixels that method detected. It establishes the ratio of true object pixels to the all pixels detected by method. Recall 
Table 1. Threshold Values for sample IR image segmentation.

\begin{tabular}{|l|l|l|l|}
\hline Image & Ostu method & Max Entropy method & StatSDM \\
\hline Animal-15 & 120 & 79 & 108 \\
\hline Animal-16 & 123 & 134 & 199 \\
\hline Animal-17 & 119 & 99 & 123 \\
\hline Human-3 & 128 & 74 & 127 \\
\hline Human-6 & 119 & 122 & 167 \\
\hline Human-7 & 118 & 131 & 137 \\
\hline Object-1 & 114 & 112 & 141 \\
\hline Object-2 & 109 & 92 & 89 \\
\hline Object-4 & 106 & 79 & 108 \\
\hline
\end{tabular}

is defined as the number of object pixels detected by the method divided by the total number of actual object pixels. It establishes the ratio of the detected pixels of object to the original pixels of object.

Let $\mathrm{A}(\mathrm{O})$ represents number of object pixels segmented by the method and let $\mathrm{A}(\mathrm{T})$ total number of actual object pixels that was physically segmented. The recall is given by equation 19

$$
\text { Recall }=\frac{A(O \cap T)}{A(T)}
$$

The precision is given by equation 20

$$
\text { Precision }=\frac{A(O \cap T)}{A(O)}
$$

Jaccard Similarity Index (JSI) [1] is used to compute overlapping of two sets. $J S I\left(G_{t}, S_{i}\right)$ represents Jaccard Similarity Index for ground truth and segmented image as per equation 21. For better segmentation result it is high and lower for it weak segmentation result.

$$
J S I(G t, S i)=\left|\frac{G_{t} \cap S_{i}}{G_{t} \cup S_{i}}\right|
$$

Dice Similarity Index (DSI) [1] is as like as JSI and lies between 0 to 1 . DSI is represented as in equation 22 .

$$
\operatorname{DSI}(G t, S i)=2 \times \frac{\left|G_{t} \cap S_{i}\right|}{\left|G_{t}\right|\left|S_{i}\right|}
$$

Absolute error rate $\left(a_{e r}\right)[1]$ is ratio of absolute error $\left(n_{e r}\right)$ to total pixels of image $(M \times N)$. For better image segmentation $a_{e r}$ is smaller and vice-versa. Absolute error, $n_{e r}$, is the difference between total segmented pixels and total ground truth pixels. The Absolute error rate, $a_{e r}$, is given by 23 .

$$
\text { AbsoluteErrorRate }\left(a_{e r}\right)=\frac{\text { AbsoluteError }\left(n_{e r}\right)}{M \times N} \times 100
$$


Table ?? shows the comparison results of segmentation behaviour for IR images. The performance is measured based on recall, precision, JSI, DSI and aer values of object detection for Ostu method, Max Entropy method and propose modified statistical method. Propose modified statistical method performs efficient than others. Also proposed method has better average error rate of as compared with Ostu method, Max Entropy method respectively.

Table 2. Comparison results of segmentation behaviour for IR images.

\begin{tabular}{|l|l|l|l|l|}
\hline Method & Parameters & Animal & Human & Object \\
\hline \multirow{2}{*}{ Ostu method } & Precision & 0.80 & 0.87 & 0.86 \\
\cline { 2 - 5 } & Recall & 1.00 & 1.00 & 1.00 \\
\hline \multirow{2}{*}{ Max Entropy method } & Precision & 0.76 & 0.86 & 0.87 \\
\cline { 2 - 5 } & Recall & 1.00 & 1.00 & 1.00 \\
\hline \multirow{3}{*}{ Ostu method } & Precision & 0.97 & 0.98 & 0.87 \\
\cline { 2 - 5 } & Recall & 1.00 & 1.00 & 1.00 \\
\hline \multirow{3}{*}{ Max Entropy method } & Absolute Error Rate & 8.42 & 5.10 & 3.18 \\
\cline { 2 - 5 } & Jaccard Similarity Index & 0.80 & 0.87 & 0.86 \\
\cline { 2 - 5 } & Dice Similarity Index & 0.87 & 0.93 & 0.92 \\
\cline { 2 - 5 } & Jbsolute Error Rate & 9.31 & 5.12 & 3.47 \\
\cline { 2 - 5 } & Dice Similarity Index & 0.85 & 0.92 & 0.93 \\
\hline \multirow{3}{*}{ StatSDM } & Absolute Error Rate & 0.58 & 0.55 & 2.35 \\
\cline { 2 - 5 } & Jaccard Similarity Index & 0.97 & 0.98 & 0.87 \\
\cline { 2 - 5 } & Dice Similarity Index & 0.99 & 0.99 & 0.93 \\
\hline
\end{tabular}

\section{Conclusion}

This work has proposed an automatic and efficient segmentation method based on statistical parameters. By combination of median and standard deviation for thresholding, automatic segmentation of IR images has been introduced. The aim of this paper to proposed an efficient image segmentation method. To achieve this aim, we proposed statistical automatic thresholding StatSDM method. The proposed method segments the IR images effectively and retrieve object from background. The experimental results illustrate that the StatSDM method attains enhanced performance for automatic object segmentation in IR images compared with global and statistical segmentation methods. These results are promising and motivated for further study to develop automatic segmentation algorithms in IR images and pattern recognition. On the other hand, proposed method could be enhanced and extended for object segmentation of former types of IR images. 


\section{Conflict of Iterest}

On behalf of all authors, the corresponding author states that there is no conflict of interest.

\section{References}

1. Jadin, M. S., \& Taib, S. (2012). Infrared Image Enhancement and Segmentation for Extracting the Thermal Anomalies in Electrical Equipment. Elektronika Ir Elektrotechnika, 120(4), 107-112. https://doi.org/10.5755/j01.eee.120.4.1465.

2. Azarbad, Milad \& Ebrahimzade, Attaollah \& Izadian, Vahid. (2011). Segmentation of Infrared Images and Objectives Detection Using Maximum Entropy Method Based on the Bee Algorithm. International Journal of Computer Information Systems and Industrial Management Applications. 3.

3. A. Duarte, L. Carrao, M. Espanha, T. Viana, D. Freitas, P. Bartolo, P. Faria, H.A. Almeida, Segmentation Algorithms for Thermal Images, Procedia Technology, Volume 16, 2014, Pages 1560-1569, ISSN 2212-0173, https://doi.org/10.1016/j.protcy.2014.10.178.

4. Heriansyah, Rudi \& Abu Bakar, Syed Ab Rahman. (2007). Defect Detection in Thermal Image using Thresholding Technique, 6th WSEAS International Conference on CIRCUITS, SYSTEMS, ELECTRONICS,CONTROL \& SIGNAL PROCESSING, Cairo, Egypt, Dec 29-31, 2007341.

5. Rogowska, Jadwiga. (2000). Overview and Fundamentals of Medical Image Segmentation. Handbook of Medical Image Processing and Analysis. 10.1016/B978012077790-7/50009-6.

6. Zhou, Mu \& Hong, Xia \& Tian, Zengshan \& Dong, H. \& Wang, Mingchun \& Xu, Kunjie. (2014). Maximum Entropy Threshold Segmentation for Target Matching Using Speeded-Up Robust Features. Journal of Electrical and Computer Engineering. 2014. 1-12. 10.1155/2014/768519.

7. Z. Delong and Z. Junbin, Minimum Error Thresholding Based on Two Dimensional Histogram, 2009 WRI World Congress on Computer Science and Information Engineering, Los Angeles, CA, 2009, pp. 169-175, doi: 10.1109/CSIE.2009.797.

8. Prof. S.T. Khandare and Prof. A. D. Isalkar, A Survey Paper on Image Segmentation with Thresholding, International Journal of Computer Science and Mobile Computing, Vol.3 Issue.1, January- 2014, pg. 441-446.

9. K. C. Markham, Comparison of segmentation processes for object acquisition in infrared images, in IEE Proceedings F - Radar and Signal Processing, vol. 136, no. 1, pp. 13-21, Feb. 1989. DOI: 10.1049/ip-f-2.1989.0003.

10. Hinojosa, Salvador \& Pajares, Gonzalo \& Cuevas, Erik \& Ortega-Sanchez, Noee. (2018). Thermal Image Segmentation Using Evolutionary Computation Techniques. 10.1007/978-3-319-63754-9_4, INTERNATIONAL JOURNAL OF SCIENTIFIC \& TECHNOLOGY RESEARCH VOLUME 8, ISSUE 10, OCTOBER 2019 ISSN 2277-8616.

11. Nawrat A., Daniec K., Warmuz T. (2013) Object Detection Using IR Camera. In: Nawrat A., Simek K., Swierniak A. (eds) Advanced Technologies for Intelligent Systems of National Border Security. Studies in Computational Intelligence, vol 440. Springer, Berlin, Heidelberg. https://doi.org/10.1007/978-3-642-31665-4_11. 
12. Morris, Nigel \& Avidan, Shai \& Matusik, Wojciech \& Pfister, Hanspeter. (2007). Statistics of Infrared Images. Proceedings of the IEEE Computer Society Conference on Computer Vision and Pattern Recognition. 1-7. 10.1109/CVPR.2007.383003.

13. Gonzalez, R.C.; Woods, R.E. Digital Image Processing, 2nd ed.; Addison-Wesley Longman Publishing Co., Inc.: Boston, MA, USA, 2001.

14. H. Deng, X. Sun, M. Liu, C. Ye and X. Zhou, Small Infrared Target Detection Based on Weighted Local Difference Measure, in IEEE Transactions on Geoscience and Remote Sensing, vol. 54, no. 7, pp. 4204-4214, July 2016. DOI: 10.1109/TGRS.2016.2538295.

15. Otsu, N., A Threshold Selection Method from Gray-level Histograms, IEEE Transactions on Systems, Man, and Cybernetics, Vol. SMC-9, No. 1, 1979, pp. 62-66.

16. .N. Kapur, P.K. Sahoo, A.K.C. Wong, A new method for gray-level picture thresholding using the entropy of the histogram, Computer Vision, Graphics, and Image Processing, Volume 29, Issue 3, 1985, Pages 273-285, ISSN 0734-189X, https://doi.org/10.1016/0734-189X(85)90125-2.

17. Kumar, Vijay \& Gupta, Priyanka, Importance of Statistical Measures in Digital Image Processing. International Journal of Emerging Technology and Advanced Engineering, Volume 2, Issue 8, August 2012.

18. Li, Z., Liu, C., Liu, G. et al. Statistical thresholding method for infrared images. Pattern Anal Applic 14, 109-126 (2011). https://doi.org/10.1007/s10044-010-01848.

19. Ulusoy, Ilkay \& Yuruk, Huseyin. (2011). New method for the fusion of complementary information from infrared and visual images for object detection. Image Processing, IET. 5. 36 - 48. 10.1049/iet-ipr.2009.0374.

20. R. Kalam and K. Manikandan, Enhancing K-Means Algorithm for Image Segmentation, 2011 International Conference on Process Automation, Control and Computing, Coimbatore, 2011, pp. 1-4, doi: 10.1109/PACC.2011.5979016.

21. T. J. Ramirez-Rozo, J. C. Garcia-Alvarez and C. G. Castellanos-Dominguez, Infrared thermal image segmentation using expectation-maximization-based clustering, 2012 XVII Symposium of Image, Signal Processing, and Artificial Vision (STSIVA), Antioquia, 2012, pp. 223-226, doi: 10.1109/STSIVA.2012.6340586.

22. Rodhouse, Kathryn Nicole, A comparison of near-infrared and visible imaging for surveillance applications (2012). Masters Theses. 6271. https://scholarsmine.mst.edu/masters_theses/6271. 